\title{
Construction Machine Pose Prediction Considering Historical Motions and Activity Attributes Using Gated Recurrent Unit (GRU)
}

\author{
Han Luo ${ }^{a}$, Mingzhu Wang ${ }^{\text {b,* }}$, Peter Kok-Yiu Wong ${ }^{a}$, Jingyuan Tang ${ }^{a}$, Jack C. P. Cheng, \\ ${ }^{a}$ Department of Civil and Environmental Engineering, the Hong Kong University of Science and \\ Technology, Clear Water Bay, Kowloon, Hong Kong \\ ${ }^{b}$ School of Architecture, Building and Civil Engineering, Loughborough University, Loughborough, \\ United Kingdom
}

\begin{abstract}
-
The variation of construction machine poses is one of the main causes for interactive on-site safety issues such as struck-by hazards. With the aim to reduce such hazards, we propose a framework for predicting construction machine poses based on historical motion data and activity attributes. After building a machine motion dataset, we develop a keypoint-based method for recognizing machine activities considering working patterns and interaction characteristics. The recognized activity information is then incorporated with historical pose data to predict future machine poses through a type of recurrent neural network (RNN), named Gated Recurrent Unit (GRU). In experiments of using excavators as the objects, our framework achieves decent performance for machine pose prediction, which is further improved by incorporating activity information, reaching an average percentage of correct keypoints (PCK) of $90.22 \%$. The results indicate the high potential of our framework in predicting construction machine poses and improving on-site safety.
\end{abstract}

\section{Keywords -}

Activity recognition, Construction machines, Construction safety, Deep learning, Gated Recurrent Unit (GRU), Pose prediction, Pose forecasting, Recurrent Neural Network (RNN)

\section{Introduction}

Construction sites are suffering from high hazard rates among all workplaces, making it a foremost concern to improve the on-site safety. According to the reports from the U.S., mainland China and Hong Kong Special Administrative Region [1-3], unsafe operation of construction machines is an essential reason of fatal hazards occurred on construction sites. In the U.S., over $38 \%$ of construction accidents are caused by interactions between construction resources (e.g. workers and machines), which also resulted in more than $16 \%$ and $29 \%$ of construction accidents in mainland China and Hong Kong SAR, respectively. Hence, it is important to monitor the motion of construction machines on sites. Typically, accidents are likely to take place when two on-site objects move towards each other, making it important for safety managers to pay attention to the changing locations (i.e. trajectories) of on-site construction resources. In recent years, several efforts have been made to automatically monitor locations of construction resources based on data captured from surveillance cameras and pre-installed devices to assist site managers with 
traditional error-prone and tedious safety inspections of construction sites [4-6]. Nevertheless, it is common but easily overlooked that accidents may still occur when the location of heavy construction machines remains unchanged, but their poses are varying constantly as deformable components of construction machine are operated. Therefore, monitoring machine poses can be a necessary supplement for safety management of construction projects.

Up till now, most site managers monitor on-site machine poses by watching surveillance videos and evaluating potential risks manually. Such manual observations of on-site safety condition are error-prone and time-consuming because they are greatly dependent on physical status and expertise of the inspector. To address such limitations, previous studies have attempted to automate pose monitoring of construction machines based on surveillance videos. For example, several efforts have been contributed to estimating past and current poses of construction machines through processing on-site videos not only using conventional computer vision techniques [7], but also further adopting deep learning techniques $[8,9]$ that have shown promising performance in many vision-based tasks. Besides, another common and practicable approach for automating pose monitoring of construction machines is processing the collected signals from pre-installed devices [10]. Most of previous studies only focus on estimating past and current machine poses which have occurred, yet understanding the current poses of construction machines is not sufficient to avoid potential hazards. Instead, pose prediction (or pose forecasting) [11], of construction machines can provide more clues to prevent possible collisions or other accidents, for which there is still a lack of research.

When tentatively investigating potential methods for predicting future poses, we found that both geometric and non-geometric information provide insights for prediction. On the one hand, future poses of construction machines are influenced by machine motions which can be informed by geometric construction data, such as the geometry information of machines as well as the construction environment. On the other hand, non-geometric construction data, such as the working task the machine is focusing on and the interaction of the machine with other objects, can provide contextual information for predicting machine poses, which is not fully considered in other research. With the aim to reduce potential on-site hazards, we propose a framework incorporating both geometric and non-geometric construction information to improve the performance of machine pose prediction, where geometric construction data refer to the dimensions (e.g. length, width, height) and coordinates of both construction machines and project terrain, while nongeometric data are semantic data such as working tasks and working natures of the construction machine. The proposed framework consists of three modules, i.e. motion capture module, activity recognition module and machine pose prediction module. Firstly, geometric construction site information is included in the machine motion capture module, and thereon historical motion data of target construction machines are provided. Next, the machine activity recognition module recognizes historical activities with the help of non-geometric construction information and historical motion data from the machine motion capture module. Lastly, the machine pose prediction module generates future poses of construction machines by integrating information of historical poses and activities. To validate the proposed framework, we adopted excavators as experiment objects as excavators own more deformable components and tend to have complex pose variations and interactions with the surrounding objects, and performed experiments based on a motion capture dataset proposed by adding historical poses data to an existing video dataset. The proposed framework is expected to provide more comprehensive information to reduce potential hazards caused by varying machine poses and to protect on-site workers.

The rest of this paper is summarized below. Section 2 reviews works related to motion analysis 
of the past and current states of construction machines, as well as machine motion prediction. Afterwards, Section 3 introduces the proposed overall framework of machine pose prediction that considers both geometric and non-geometric construction information, including detailed descriptions and implementation approaches of each framework module. Next, taking excavators performing earthmoving tasks as the example, the proposed overall framework is verified in Section 4, and thereon discussion and insights about the experiment results are given. In the end, conclusion is stated in Section 5.

\section{Related Works}

Machine motion (i.e. locations, poses and movements) monitoring has attracted increasing attentions of the construction industry in recent years because of the high on-site hazard rates resulted from moving construction machines. On construction sites, machine motion monitoring includes not only analyzing machine motions that have occurred in the past and current time, but also, on this basis, predicting potential motions of construction machines in the future. Therefore, this paper firstly reviews studies about on-site machine motion analysis, followed by the methods related to on-site machine motion prediction.

\subsection{On-site machine motion analysis}

Machine motion analysis includes many tasks such as machine localization, pose estimation, and activity analysis, which provides rich information of the past and current motions for on-site safety management. In the past, a common practice was to manually analyze machine motions, which requires inspectors to visit the construction site or watch surveillance videos captured from the site. Under this circumstance, it is necessary for the inspectors to intensively concentrate on identifying potential hazards based on their experiences, which, however, is error-prone after inspectors working for a long time. Consequently, automated on-site machine motion analysis is in need.

Machine localization. Various pre-installed devices have been widely applied to automatically localize construction machines on sites. For example, Global Positioning System (GPS) [12], Radio Frequency Identification (RFID) [13,14] and Ultra-Wideband System (UWB) [15] have been utilized for real-time localizing construction machines. The high precisions of such methods make them still play a significant role in the positioning of objects on construction sites. However, such methods usually suffer from complicated installations and lack visual information. In addition, a priori knowledge of the mapping between pre-installed localization devices and construction machines must be obtained in order to distinguish the localization signals. With the large number of surveillance videos available, computer vision is another effective way of localizing on-site construction machines with a wealth of visual information. Based on the videos captured from surveillance cameras installed on construction sites, Chi and Caldas [16] utilized background subtraction for automatically identifying the positions of construction machines. However, this technique is not able to localize immobile construction machines and distinguish the types of construction machines. To address such limitations, support vector machines (SVMs) was studied [4]. But such method requires careful design of complex handcrafted features for interpreting surveillance videos and images. The manual designed features will influence the accuracy of machine localization. Afterwards, deep neural networks, such as region-based fully convolutional network (R-FCN) [6] and the single shot detector (SSD) [17], have been applied to enhance the 
performance of computer vision in localizing construction machines from images and videos with significant high accuracies. However, accidents still happened when heavy construction machines stay in the same location but working with varying poses. Therefore, knowing locations of different types of construction machines is inadequate for reducing potential hazards.

Machine pose estimation. Estimating poses of construction machines gradually attracts more attention in the process of machine motion analysis. For instance, angular sensors, such as Inertial Measurement Units (IMUs) [18-20] and accelerometer-based joint angle sensors [21-23] have been deployed to measure the partial poses of excavators and cranes. Nevertheless, such angular sensor -based methods only estimated the poses of construction machines independently without obtaining global positions relative to the construction site. To address the limitation, real-world positions of machine joints are estimated in later studies by leveraging Global Positional System (GPS) [24,25] and Ultra-Wide Band (UWB) [10,26]. Existing studies have demonstrated that preinstalled devices can provide satisfactory results of pose estimation, but such methods lack visual information and installing pre-installed devices is labor-intensive and time-consuming. As surveillance videos are available on many construction sites currently, vision-based techniques are another alternative for automatically estimating poses of construction machines. Soltani et al. [7] have tried to identify partial poses (i.e. booms and arms) of construction machines by combining $\mathrm{k}$-means algorithm and background subtraction. Later, deep learning techniques were adopted to automatically obtain partial poses (i.e. bucket, stick and boom) of articulated construction robots [8] and full body poses of construction machines [9]. It is found that methods based on data from pre-installed devices or surveillance cameras have their own advantages and disadvantages. Preinstalled devices have low cost and can transfer the on-site signals steadily without much influence from environmental changes. The main limitation is that frequent purchase and installation of new devices may be needed since construction machines often changes and pre-installed devices are fragile in complex construction environment. On the other hand, vision-based approaches are capable of providing rich visual information of the construction environment, but they are susceptible to the surrounding conditions such as light conditions and heavy occlusions.

Machine activity analysis. On the basis of object localization and pose estimation, machine activity analysis can provide contextual information for safety management and productivity management. Activity analysis of construction machines can be divided into two categories, i.e. single machine activity analysis and multiple machine analysis. For single machine analysis, kinematics models are often adopted to recognize activities of the target machine by considering velocity and acceleration of the machine joints when precise 3D geometry information of the machine is known [27,28]. Since on-site machines may be different at each stage of the construction projects, it is unrealistic to measure such accurate information of all involved heavy machines. A more general method of single machine activity analysis is using statistical features [20,29], which can be applied to both IMU data and video data. It finds effective time-domain and frequency-domain features after removing irrelevant and redundant information, and then compares similarity between the target sequence and the standard sequence based on the extracted features. Such studies can only be performed after all data were collected, which means that realtime analysis is not supported. As for recognizing activities of multiple construction machines in one frame, machine learning algorithms of detection, tracking and activity recognition were adopted in combination to categorize machine activities without detailed information of the target machines [30,31]. Such methods usually train each machine learning algorithm separately by a distinct style of training labels, and even build a specific model for each construction machine in order to achieve better performance, which however requires a large amount of training data for 
each machine learning module and cost much computational power during the whole training process. To reduce the dependence of training data and the cost of computational power, interaction analysis can be a useful and effective strategy [32], which considers the coexistence, proximity and action consistency between construction machines. But these studies need to optimize the threshold proximity according to their datasets, which hinders such method to be more generalized. As suggested by [29], activities of construction machines can be broken down into four levels from coarse (one type) to fine (four types) when the machine engine is on. The existing interaction analysis -based method [32] can only recognize two types of activities (idle and busy), which is inadequate for more precise management for on-site safety or productivity. Therefore, a better strategy is required for analyzing interactive natures of construction machines and recognizing more precise types of activities. Such strategy should have the following characteristics: (1) can be adopted with relatively high accuracy without precise geometry information of all involved construction machines; (2) can be performed in real-time mode i.e. when construction machines are working; (3) can be generalized to construction machines working with various types of activities in different construction environment.

\subsection{On-site machine motion prediction}

In addition to localizing machines and estimating their poses, predicting motions of construction machines, such as location changes and pose variations, can further facilitate safety management by knowing the potential hazards in advance. Focusing on moving machines, motion prediction generates the most likely locations and poses of the target in the long-term or short-term future, given the prior knowledge of their past motion.

Previous studies have attempted to structure priori motion model, especially Kalman filter [33], for predicting $2 \mathrm{D}$ future locations of moving objects. Nevertheless, such methods require precise calibration of a lot of parameters to obtain good prediction results. Under this circumstance, Alejandro et al. [34] proposed a method of object motion prediction by learning typical motion patterns through pairwise clustering, which reduced the requirements of precise parameter calibration. This method firstly clustered the input sequences based on predefined typical moving patterns of the target object, and then predicts the most possible trajectory cluster by maximum likelihood estimation according to the mean value and standard deviation of all clusters. Afterwards, a particle filter algorithm [35] was developed with the ability to predict long-term future locations of moving vehicles in a large unstructured environment. Through the vehicle state equation and the constraints provided by the surrounding environment attributes, this algorithm could predict the vehicle locations considering the uncertainty of the environment and the stopping probability of the target vehicle. But predicting $2 \mathrm{D}$ locations is insufficient when objects change their locations in 3D spaces. Consequently, a real-time 3D object location prediction network is developed in [36] by structuring sequence-to-sequence architecture based on long short term memory (LSTM) units. Compared with previous efforts in 2D location prediction, this LSTMbased method utilized the correlation between input sequences to predict higher dimensional information without losing accuracy, which shows the promising performances of deep learning methods in time-series prediction tasks.

The studies mentioned above only concentrated on locations changes of the whole target object. When the main body of construction machines stays still at one location, predicting their pose variation in the future time frame is more important to prevent possible safety issues. According to [9], poses of construction machines can be represented by keypoints, which is similar to human 
poses. Intuitively, learning historical keypoint-based poses of construction machines will provide heuristic clues for predicting their poses in the long-term and short-term future. However, there is a lack of studies on predicting poses of construction machines with multiple deformable components. As inspired by studies on human pose prediction [11,37], based on pose information extracted from complex images or other data sources, recurrent neural network (RNN), a deep learning method for processing time-series data, has shown great performances in learning spatiotemporal characteristics from input sequences and thereby obtaining reliable results in shortterm prediction without the manual design of motion patterns. However, in comparison to studies on human motion prediction that usually predicts human poses when the individual performs a single activity, machine motion prediction involves poses across several different activities when the target machine is completing a task. In this case, RNN considering contextual information, such as activity types, will be a potential solution of on-site machine pose prediction.

\section{The Proposed Framework for Construction Machine Pose Prediction}

The overall framework of machine pose prediction considering non-geometric construction information is illustrated in Figure 1. Firstly, the motion capture module is to obtain historical motion data (i.e. locations, poses and movements) by processing geometric construction information captured by external devices such as surveillance cameras and pre-installed devices. Subsequently, the machine activity recognition module provides historical activity information considering both historical motions and non-geometric construction information such as working tasks and working natures. The adopted non-geometric construction information is the documented information of construction projects, or construction knowledge and conventions summarized by experienced practitioners. It should be emphasized that such information should have correlations with activity types, moving patterns, operation cycles and interactive objects of construction machines, which can provide clues to distinguish different machine activities. Eventually, historical motions and activities information are incorporated as the input to the machine pose prediction module, which generates future poses of construction machines. 


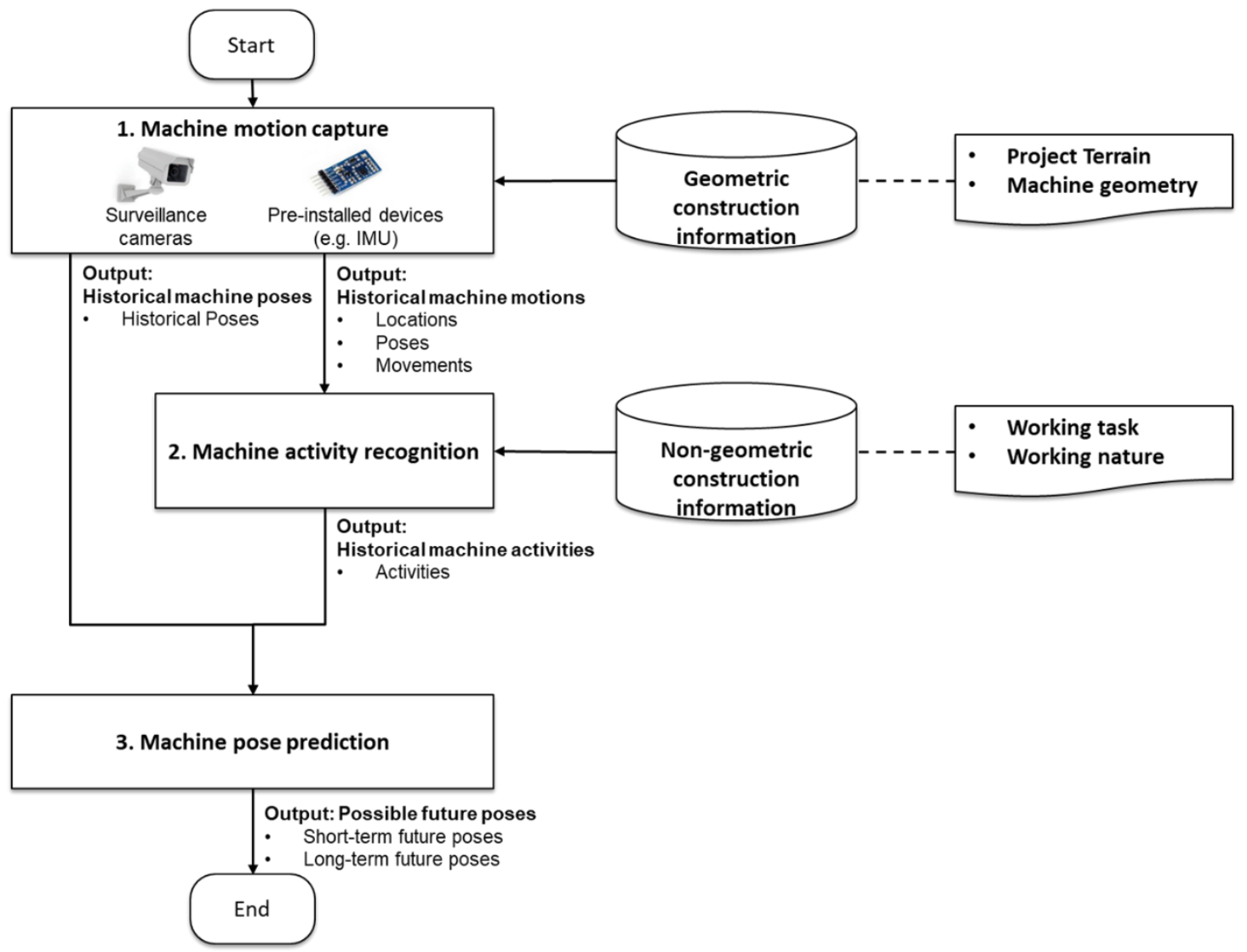

Figure 1. The overall framework of machine pose prediction considering non-geometric construction information

\subsection{Motion capture of construction machines on construction sites}

Motion capture module is the foundation of pose prediction, which aims to obtain motion data (i.e. locations, poses and movements) of all moving machines. Given an interested construction site, a set of surveillance cameras or pre-installed devices (e.g. IMUs and GPS) can be deployed to collect visual or non-visual signals of on-site geometry information depending on the practical requirements of a monitoring task. As pre-installed devices or surveillance cameras are complementary considering their advantages and limitations, they can be adopted individually or together depending on the conditions of working environments and the requirements of construction projects. Since this paper focuses on the machine pose prediction enhanced by activity recognition, motion capture techniques are not discussed. Throughout this paper, motion data (i.e. locations, poses and movements) of each machine have been captured for activity recognition and machine pose prediction, unless otherwise specified. Motion data can be interpreted by processing the collected signals using different algorithms depending on the purpose. Object detection models such as Faster R-CNN [38] can be used for detecting machine locations while object tracking models such as Tracking-learning-detection [39] can be applied for machine tracking. In terms of machine keypoints, methods are proposed based on deep learning models for human-pose estimation models such as Cascaded Pyramid Network [40]. The methods for estimating machine keypoints are trained using data containing the coordinates of each keypoint of construction 
machines. More details of the three types of motion data are explained in the following.

\section{(1) Locations}

In this paper, a location is a particular place or position occupied by a construction machine, which suggests the presence of a machine on the interested construction site. When processing images captured by surveillance cameras, the location of a machine is often indicated by a bounding box in the image, which represents the relative coordinate with the adopted surveillance camera as the reference system. When processing non-visual signals from pre-installed locating devices like GPS, the location is represented by the global coordinate of the locating device.

\section{(2) Poses}

Following our previous work [9], poses of construction machines can be represented by their skeleton keypoints. Keypoints should be defined in an 'object-centric' way and follow three basic rules, which are: (1) defining basic keypoints according to major joints of the machine design; (2) annotating supplementary keypoints that are in the intersections between the machine body and its working environment; (3) full body poses of each construction machine can be formed by connecting all the annotated keypoints. For example, keypoints of typical construction machines such as excavators and trucks are shown in Figure 2.

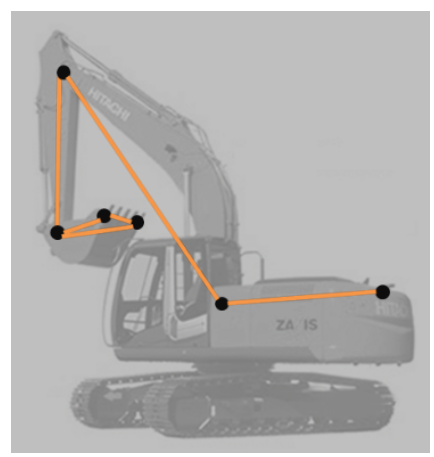

(a) Excavator keypoints

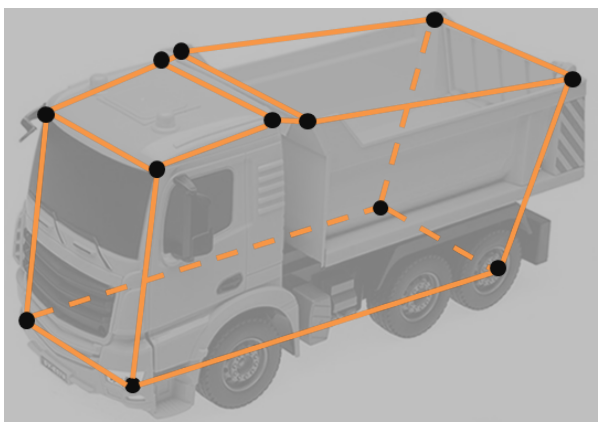

(b) Truck keypoints

Figure 2. Pose keypoints of typical construction machines

\section{(3) Movements}

Locations and poses only represent the states of a machine at a certain time. When continuously monitoring construction sites, information about variations of machine locations and poses over a period of time is more useful. Thus, the movement data (i.e. trajectory) is obtained by matching objects and their skeleton keypoints at each discrete time points throughout a specific time period. The movement data ultimately generates both location trajectory and keypoint-based pose trajectory of the machine.

\subsection{Activity recognition of construction machines considering non-geometric construction information}

For different types of on-site activities, different construction machines are commonly operated in the form of various poses with certain moving patterns. Knowing the activity type can provide contextual information for understanding the pose moving patterns, which in the end, can facilitate predicting machine poses. Therefore, this study first recognizes the activity types and uses the activity information as prior knowledge to assist with the machine pose prediction. 
Generally, it is observed that construction machines operate in different poses with certain patterns during different activities, making it possible to leverage keypoint and pose information for recognizing excavator activities. As inspired by previous studies [32], only relying on motion data is insufficient for accurately recognizing activities of construction machines, since nongeometric construction information such as working tasks (e.g. earthmoving task) and working natures (e.g. possible machines to interact with) also has influence on the activity recognition results. One typical example is that the status of a truck should be classified as working when it is interacting with an excavator in an earthmoving operation, even though the truck location remains unchanged. Non-geometric information can provide more contextual knowledge to understand the activities performed by the machine. For example, under a certain working task, there can be several typical activities operated by different machines, and each of them has their own patterns as well as interactions with each other. Such prior knowledge can be leveraged to assist with the activity recognition. Hence, both motion data and non-geometric construction information are leveraged in our activity recognition module to recognize machine activity types and generate historical activity information.

\section{(1) Activity recognition methodology}

As earthmoving operations are the fundamental construction tasks of construction projects and excavators is an essential machine of this process with most deformable components, in this paper, excavators performing earthmoving tasks are utilized as examples of construction machines. Although there have been some approaches developed for recognizing machine activity [30,31], most of them rely on deep learning which require large amount of annotated data for training the model. We propose a methodology based on pose keypoints for excavator activity recognition based on both motion data and non-geometric construction information. Our proposed method is intuitive and effective, without the requirement of pre-training the model on large datasets. It is noted that our overall framework for pose prediction is generalizable, so other activity recognition approaches can also be applied to obtain the activity type and assist with pose prediction.

During earthmoving tasks, when a dump truck stop at a location and is ready for the excavator to perform filling activities, excavators often interact with dump trucks with repetitive operational cycles (i.e. "Dumping" $\rightarrow$ "Swinging" $\rightarrow$ "Loading bucket" $\rightarrow$ "Swinging" $\rightarrow$ "Dumping", as shown in Figure 3) [31,41]. To distinguish those activities of the excavator straightforward, the status of the dump truck that interacts with the target excavator is considered. Intuitively, when performing "dumping" and "loading bucket" activities, an excavator often exhibits similar moving patterns, i.e. it rotates its bucket back and forth with the main body remain stationary. Under such circumstances, the dump truck remains still, hence the distance between main body of the excavator and the location of the dump truck theoretically will not change. Figure 4 illustrates the top-down view of the relative positions of the target excavator and the dump truck that it interacts. It is noticed that, during "dumping", the target excavator releases soils or rocks from its bucket at a location above the dump box of the truck. By contrast, when the excavator is "loading bucket", the position of the excavator bucket is outside the unloading area of the truck. For "swinging" activities, a typical observed moving pattern of the excavator is that it is likely to freeze most of its deformable components (e.g. boom and arm) and rotate as a rigid body along its rotation axis. Apart from the above-mentioned repetitive activities occurred in the earthmoving operational cycles, an excavator usually remains idle when waiting for a full dump truck to be replaced by an empty one, during which all the deformable components of the excavator keep unmoved without any rotations and translations. Based on the above observations, the characteristics of excavator pose variation, and interactions between excavators and trucks are utilized for recognizing 
excavator activities.

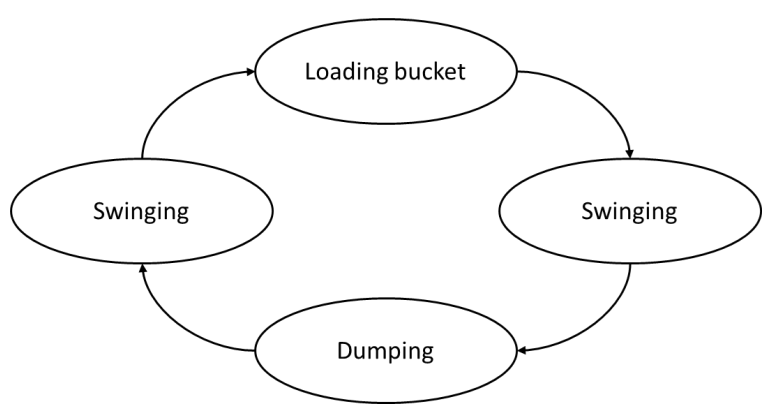

Figure 3. Repetitive operational cycles of an excavator when interacting with dump trucks [31]

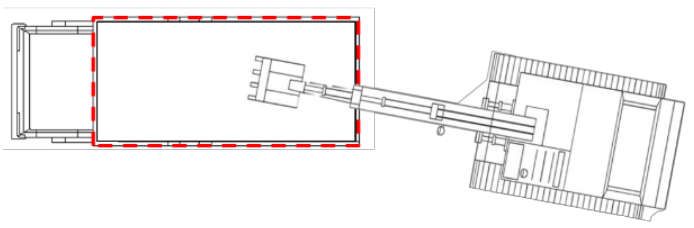

(a) Dumping
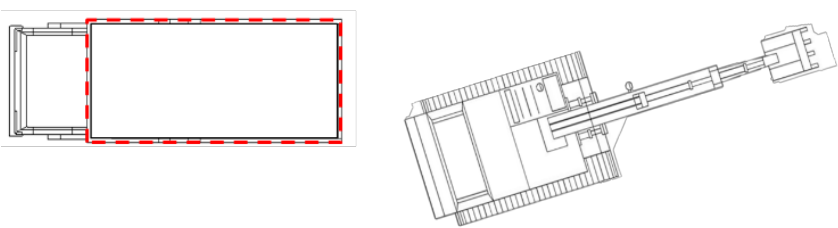

(b) Loading bucket
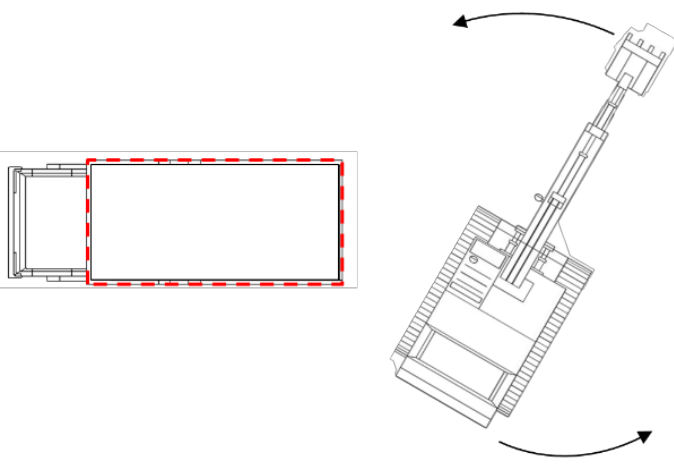

(c) Swinging

Figure 4. Top-down view of the relative relationship between the excavator bucket and the dump box of the truck during different activities, where the red dashed line represents the dump box

Figure 5 illustrates the process of activity recognition when either one-to-one interaction or group interactions of construction machines exist during earthmoving tasks. Note that working without interactions is beyond the focus of this study. Based on the information of machine types, locations, poses and movements extracted from historical machine motion data, our methodology considers several factors including co-existence, proximity and working patterns of machines. 


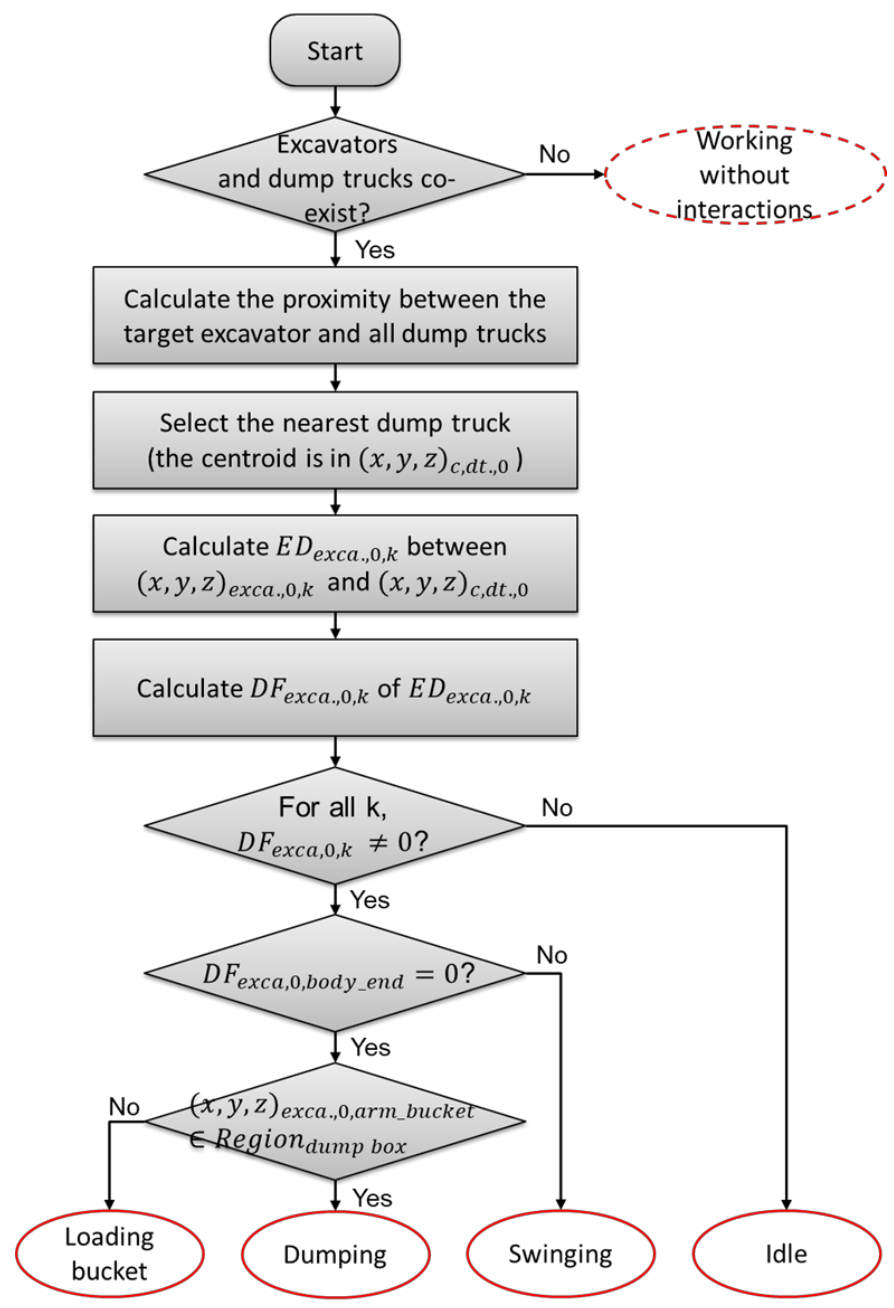

Figure 5. The proposed activity recognition methodology

Firstly, the proposed activity recognition methodology determines the co-existence of excavators and dump trucks in the scene through checking their location data obtained from the Motion Capture Module. If multiple excavators exist, the proposed methodology will select the onsite excavator one by one as the target excavator, and then apply the remaining steps for each selected excavator. If more than one dump truck exists on the monitoring site, our method will select the nearest dump truck to the excavator as the interactive machine considering proximity relationships among construction machines. In this step, the distance between the centroid of the excavator and the centroid of each dump truck is calculated through Equation (1) and (2), where Equation (2) calculates the centroid coordinates of each machine by averaging the positions of its keypoints. Then, the dump truck with the smallest distance to the excavator is selected as the nearest one.

$$
\begin{gathered}
\text { Distance }=\sqrt{\left(x_{1}-x_{0}\right)^{2}+\left(y_{1}-y_{0}\right)^{2}+\left(z_{1}-z_{0}\right)^{2}} \\
x_{c, i, j}=\overline{x_{i, j, k}}, \quad y_{c, i, j}=\overline{y_{i, j, k}}, \quad z_{c, i, j}=\overline{z_{i, j, k}}
\end{gathered}
$$


where,

$\left(x_{0}, y_{0}, z_{0}\right)$ and $\left(x_{1}, y_{1}, z_{1}\right)$ : two positions need to calculate the Euclidean distance;

\section{$\mathrm{x}$ : the $\mathrm{x}$-axis position;}

$\mathrm{y}$ : the $\mathrm{y}$-axis position;

$\mathrm{z}$ : the $\mathrm{z}$-axis position;

c: the centroid of each machine;

$\mathrm{i}$ : the type of the target construction machine, which can be exc. (excavators) or dt. (dump trucks);

$\mathrm{j}$ : the identification number of a construction machine appeared on the construction site;

$\mathrm{k}$ : each pose keypoints of each construction machine.

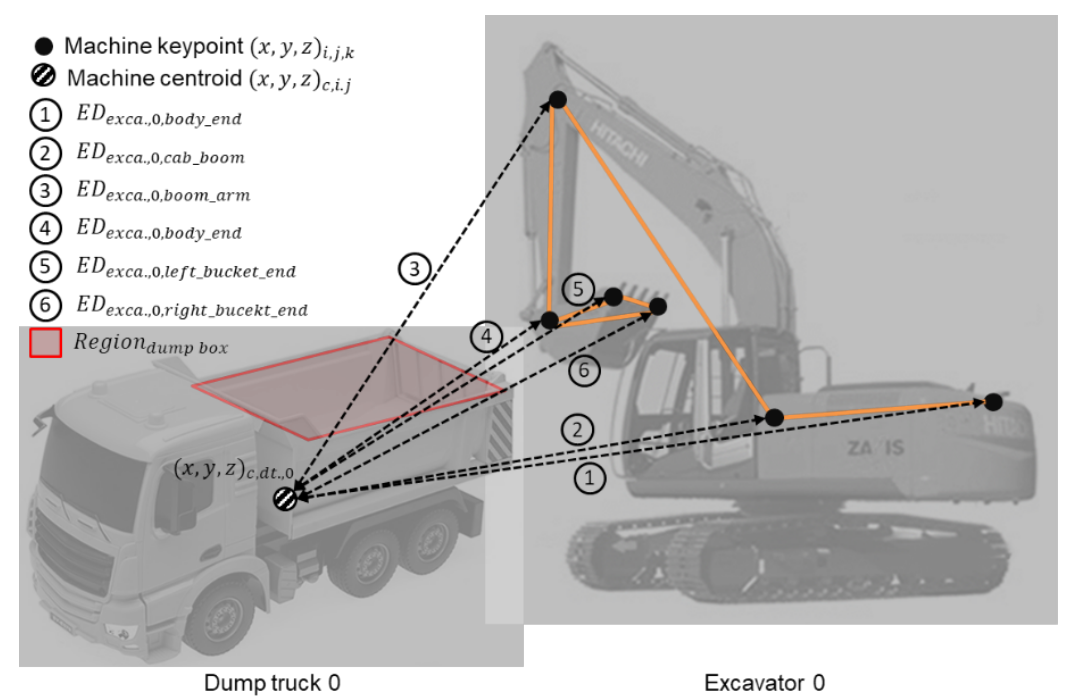

Figure 6. Illustration of the physical meanings of variables adopted in the proposed activity recognition methodology

After the interacting dump truck is determined, as shown in Figure 6, the Euclidean distance $\left(E D_{\text {exca., }, k}\right)$ between each pose keypoint of the excavator $(x, y, z)_{\text {exca., }, k}$ and the centroid of the dump truck $(x, y, z)_{c, d t ., 0}$ is computed by Equation (1), where $\mathrm{j}=0$ represents the ID of the target excavator and the dump truck it interacts. Then, the first order difference $\left(D F_{\text {exca., }, k}\right)$ of Euclidean distance $E D_{\text {exca., }, k}$ is obtained through Equation (3).

$$
D F_{\text {exca., }, k}(t)=E D_{\text {exca., },, k}(t+1)-E D_{\text {exca., },, k}(t)
$$

where,

$E D_{\text {exca., }, k}(t)$ and $E D_{\text {exca., }, k}(t+1)$ : the values of Euclidean distance at time $\mathrm{t}$ and $\mathrm{t}+1$; $D F_{\text {exca., }, k}(t)$ : the first order difference of $E D_{\text {exca., }, k, k}$ at time $t$.

When the first order differences of the Euclidean distance between all the excavator keypoints and the truck are zero, the target excavator does not have any translational and rotational motion, which indicates that the excavator is idle. Otherwise, the excavator is performing other activities, which can be "dumping", "loading bucket" and "swinging". As discussed above, the main body 
and the bucket of the excavator show different spatial relationships with the truck during different activities in the operational cycles. Based on such characteristics, we use the location changes of machine keypoints over a period of time to understand the spatial relationship among machines and help with recognizing the activity type. The movements of keypoints are analyzed based on time series data, i.e. the coordinates of each keypoint across consecutive time frames. Compared to Cab_boom keypoint that has small motions during earthmoving tasks, Body_end keypoint is more suitable to illustrate movements of the main body. If the distance between Body_end keypoint and the centroid of the dump truck stably decreases or increases, the excavator is swinging, as shown in Figure 4 (c). Otherwise when the distance remains a constant value, movements of the bucket should be considered to further discuss the activity of the target excavator. To express movements of bucket, Arm_bucket keypoint is adopted because it is visible in more cases compared with the two keypoints at the bucket boundary. In the case that the Body_end keypoint is not moving, the excavator activity is recognized as "dumping" if the Arm_bucket keypoint is inside the dump area, while the result is "loading bucket" if the Arm_bucket is outside, as shown in Figure 4 (a) and (b).

\section{(2) Pre-processing of motion data and post-processing of recognition results}

The proposed methodology is very intuitive and easy to be implemented. Nevertheless, in real applications, motion capture devices, transmission tunnels and processing algorithms may lead to noisy data which is likely to affect the accuracy of proposed method for recognizing machine activities. In addition, human operators may not have the exact same operations each time, which can introduce uncertainties to the machine movement pattern and mislead the proposed method. To reduce the influences of noisy motion signals and human uncertainties, both pre-processing and post-processing techniques are applied.

Wavelet transform smoothing for pre-processing. Wavelet transform [42] is utilized to smooth the captured motion data. Compared to Fourier Transformation that is another common noise smoothing localized only in frequency domain, wavelet transform employs wavelets and realizes time-frequency transforms, which is more appropriate for processing unstable signals like machine motion data. Afterwards, the smoothed data are used by the proposed methodology to recognize activities of excavators. However, sometimes the captured motion data will fluctuate intensively because of uncertainties from machine operators, motion capture devices or algorithms, in which the noise cannot be handled by wavelet transform. In this case, incorrect recognition of machine activities still exists. Therefore, a post-processing mechanism is utilized to compensate misrecognition errors and optimize activity recognition results.

A rollback mechanism for post-processing. As discussed above, a rollback mechanism is designed to reduce uncertainties resulted from machine operator and other unexpected sources. The rollback mechanism is based on majority voting and sliding window techniques. First of all, the per-frame activities are recognized independently by the proposed activity recognition methodology. As illustrated in Figure 7, the recognized activities in the past time window (w) will be extracted once a new stream of motion data is captured. Then, majority voting is applied to the recognized activities for the frames within the time window (w). To make this process more accurate, the overlap between every two neighbor time windows is considered by the time step (s). If the voted activities of two neighbor time windows are different, frames in first half of the overlap area will be assigned with the voted activity of the former time window. The frames in another half will be assigned with the voted activity of the later time window. 
Do not consider time window overlap:

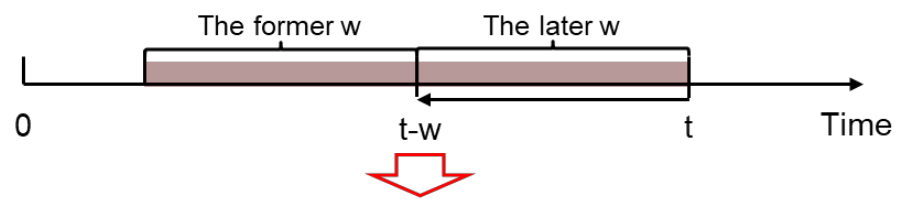

Consider time window overlap:
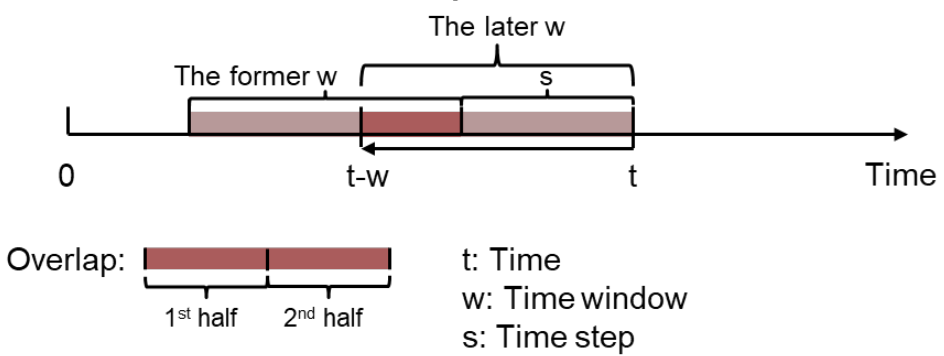

Figure 7. An illustrative diagram of the rollback mechanism

\subsection{Machine pose prediction}

Machine pose prediction is the core of this research. Based on the first module (machine motion capture) and the second module (machine activity recognition), time-series data including data of poses (represented by features of keypoints) and their corresponding activity types are integrated by adding the numerical representation of activity type into the keypoint feature vector. Then, the newly created keypoint feature vectors of all the keypoints are fed into the last module for machine pose prediction. This module learns and extracts typical features of the historical poses along with the information of different activities performed by the machine. Finally, based on the learned clues, the machine pose prediction module generates short-term or long-term future poses of the machine so as to indicate the potential area occupied by the machine and provide more comprehensive information for safety management.

In this research, we propose a method using GRU (gated recurrent unit) for machine pose prediction. GRU [43] is a common type of RNN, which is used for processing time-series data and learning complex dynamic temporal features from the input sequences. Compared to traditional RNNs which are difficult to capture long-term dependencies, GRU tries to solve the problems of long-term memory and gradients in backpropagation through gating units inside, which is similar to the design of the long-short term memory (LSTM) unit, a popular solution of sequential data processing. As indicated by [44], GRU demonstrates good performances similar to LSTM, but requires less computational power with higher efficiency. Therefore, GRU is applied, of which the illustrative diagram is shown in Figure 8. Within a gated recurrent unit, there are two gates to control and update the state of the GRU model, i.e. update gate $\left(z_{t}\right)$ and reset gate $\left(r_{t}\right)$. These two gates build a mechanism to determine which part of the input should be kept or abandoned. For each element in the input time-series data, GRU computes functions from (4) to (7). Based on this mechanism, GRU performs well in time-series related tasks.

$$
\begin{aligned}
& r_{t}=\sigma\left(W_{r} \cdot\left[h_{t-1}, x_{t}\right]\right) \\
& z_{t}=\sigma\left(W_{z} \cdot\left[h_{t-1}, x_{t}\right]\right)
\end{aligned}
$$




$$
\begin{aligned}
\widetilde{h_{t}} & =\tanh \left(W \cdot\left[r_{t} * h_{t-1}, x_{t}\right]\right) \\
h_{t} & =\left(1-z_{t}\right) * h_{t-1}+z_{t} * \widetilde{h_{t}}
\end{aligned}
$$

where,

$x_{t}$ : the input sequence of a gated recurrent unit at time t;

$h_{t-1}$ : the hidden states at time t- 1 or the initial hidden state at time 0 ;

$h_{t}$ : the hidden states at time $\mathrm{t}$;

$\widetilde{h_{t}}$ : the new memory concludes the features of newly input sequence $x_{t}$ and the hidden states at time t-1, $h_{t-1}$;

$\sigma:$ the sigmoid function;

*: the element-wise product;

W: trainable parameters.

As demonstrated in Figure 9, in the GRU-based machine pose prediction pipeline, the sequence of a number of preceding keypoint feature vectors is the input of GRU model. After processing by the GRU mechanism, the prediction result of machine poses in the future frames will be generated. For the model used in this study, one layer of bidirectional GRU with hidden size of 256 as the RNN cell number is adopted to more precisely extract temporal features in both forward and backward directions. To output the final prediction result of the machine poses, two fully connected layers are utilized following the GRU model. One of the fully connected layers has a hidden size of 256 followed by a ReLU nonlinearity layer. The other has a hidden size that equals to the number of all keypoint coordinates, which directly exports the coordinates of the future poses of the target construction machine. 


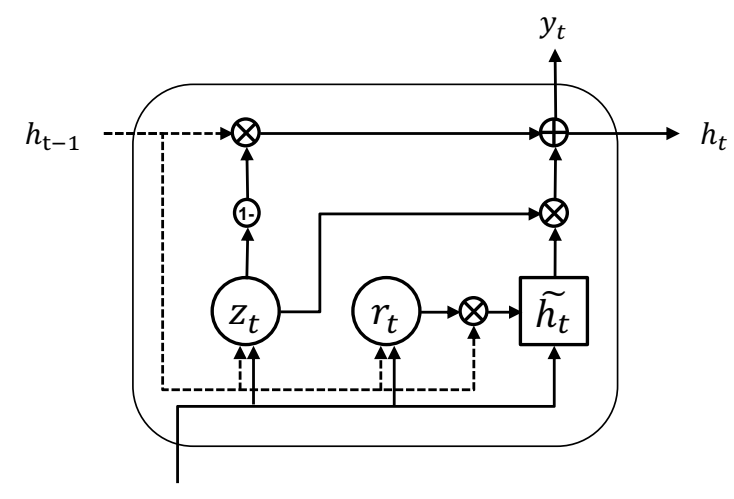

Figure 8. Illustration of gated recurrent units (GRU) [44]

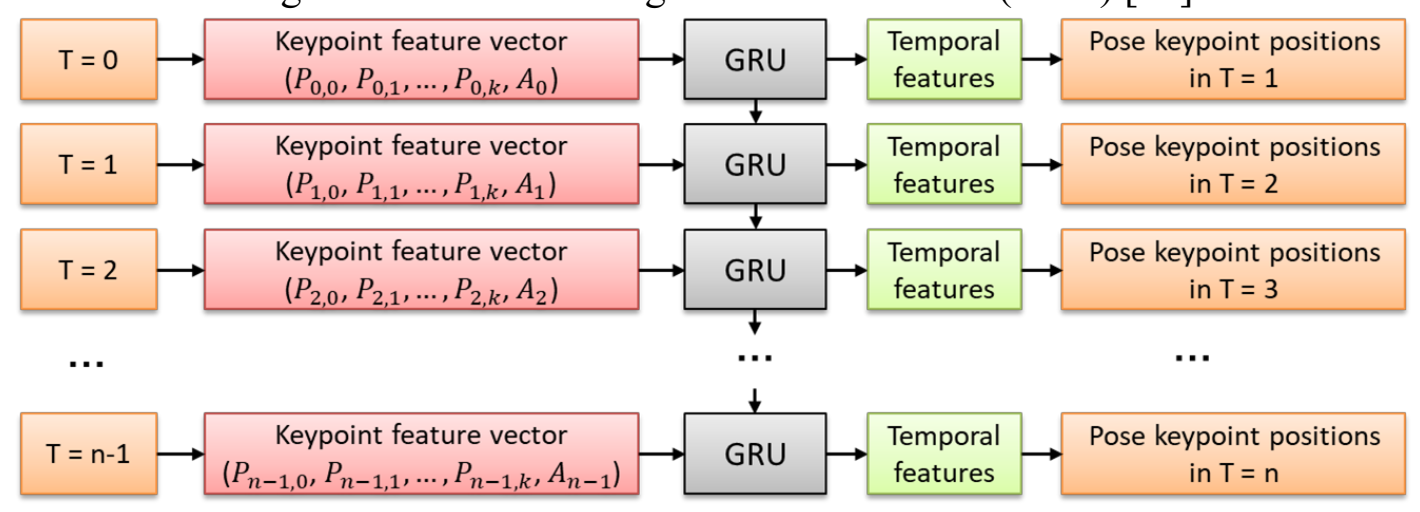

$P_{t, k}$ : the coordinates of the $\mathrm{k}^{\text {th }}$ pose keypoint of the target machine when the time $\mathrm{T}$ is $\mathrm{t}$. $A_{t}$ : the activity type performed by the target machine when the time $\mathrm{T}$ is $\mathrm{t}$.

Figure 9. Illustration of the GRU-based machine pose prediction process

\section{Validation of the Proposed Framework}

To validate the feasibility and effectiveness of the proposed framework for machine pose prediction, excavators are adopted as the experiment objects. One reason is that excavators are fundamental and essential machines in construction projects with the most deformable components. Besides, excavators tend to have more complex pose variations and interactions with the surrounding objects.

\subsection{Motion capture data collection}

For the reason that there is a lack of dataset for machine pose prediction, a motion capture dataset is created on the basis of the videos published by [31]. All the videos are filmed at $25 \mathrm{fps}$ (frame per second) with a size of 480 pixel*272 pixel from various civil and building projects, where excavators and trucks have a significant amount of interactions during working operations. Activity recognition and pose prediction based on this dataset are challenging because the adopted videos vary a lot from each other in terms of illumination and camera distance. Additionally, there exist difficult situations for recognition or prediction where components of machines are temporarily occluded by soils, co-existed machines, and even their own bodies. Originally, there are bounding box and activity annotations for each machine in the provided videos. To prepare the 
dataset for machine pose prediction using keypoints, annotations of construction machine pose keypoints are supplemented according to our previous work [9]. Since video data only have the information of $\mathrm{x}$ and $\mathrm{y}$ axis with the same values in $\mathrm{z}$ axis (i.e. $\mathrm{z}=0$ in video data), the coordinates of keypoints $\left(P_{t, k}\right)$ is represented by $2 \mathrm{D}$ coordinates, i.e. $(\mathrm{x}, \mathrm{y})$, in our experiments. It is known that the pose variations of trucks are much simpler than other machines during working. Therefore, we directly use bounding box annotations of trucks as keypoints of poses in this experiment to simplify pose estimation of trucks. For excavators, each piece is labelled with six keypoints, and one example is shown in Figure 10. Pose annotations are created using the CVAT annotation tool [45] in .xml format. Ultimately, the motion capture dataset proposed in this paper have five videos containing 53,484 frames labelled with bounding boxes, activities and poses. The number of frames of each adopted video is listed in Table 1.

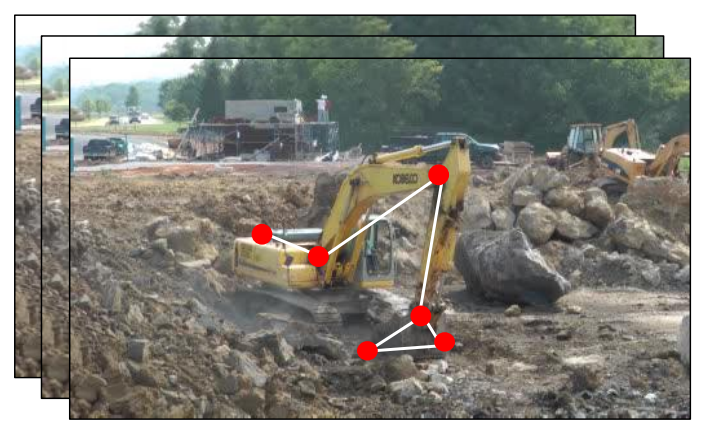

Figure 10. An example of excavator pose annotation based on keypoints in our dataset

Table 1. Total frames of each video in the dataset

\begin{tabular}{cc}
\hline Video ID & Total Frames \\
\hline 104154 & 16419 \\
134516 & 15864 \\
150625 & 3808 \\
152129 & 6012 \\
142932 & 11381 \\
Total & 53484 \\
\hline
\end{tabular}

\subsection{Implementation details}

The experiments environment is Ubuntu 16.04 operating system with an Intel Core i7-6700 CPU@3.40 GHz ×8, a NVIDIA GeForce GTX 1080 GPU. The experiments are conducted using Python and they are mainly based on Pytorch [46], which provides libraries for constructing GRU models and increasing processing speed.

On the basis of the proposed motion capture dataset of excavators, two experiments are firstly performed to separately test the performances of the second and third module of our machine pose 
prediction framework, which considers non-geometric information.

The second module, machine activity recognition, is tested and evaluated using all the frames in which excavators and trucks have interactions during earthmoving tasks on the shooting construction sites. Per-frame activities are recognized using the proposed activity recognition methodology. For the convenience of conducting experiments, the size of the sliding window is one second ( 25 frames) which equals to the frames captured per second for the on-site videos. The time step when considering the overlap between sliding windows is the result that rounds up the half length of the sliding window (i.e. 13 frames).

While for the third module, machine pose prediction, $70 \%$ of the proposed dataset is used as the training set for training the machine pose prediction model, $10 \%$ are used as the validation set for choosing a model with the highest accuracy, and the rest $20 \%$ are used as the test set for evaluating the model performance. When purely testing the performance of this module, we directly use the ground-truth activity information for creating the input keypoint feature vectors for training, validation and testing. To keep consistent with the common practice in [11], during each training iteration, the keypoint feature vectors of the past two seconds (50 frames in this experiment) in the video are used as the input sequence for the machine pose prediction model, after which the short-term machine poses in the future 0.4 seconds ( 10 frames) will be predicted. In the process of training the machine pose prediction model, L2 function is adopted as the loss function to calculate the training loss, i.e. the error between the predicted coordinates of construction machines and the actual values. The optimizer is Adam algorithm [47] because it requires less computation power and it is convenient to be implemented.

In the end, the outputs from the second module along with the motion data are used as the input for the third module, to evaluate the performance of the overall framework on the test dataset. Instead of using ground-truth activity information as part of the input for testing the third module alone, the recognized activities from the machine activity recognition module are added to original keypoint feature vectors to create new ones. Then, without extra training processes, the overall framework outputs short-term future poses of construction machines of the test set through directly adopting the trained GRU-based machine pose prediction model from the third module. Therefore, the metrics of the overall framework are the same as those for evaluating the third module separately.

\subsection{Evaluation metrics}

\subsubsection{Activity recognition metrics}

Accuracy and row-normalized confusion matrix are two approaches for evaluating the outputs of activity recognition in this paper.

Accuracy. Accuracy is one metric commonly used for evaluating classification tasks. As illustrated in Equation (8), it is the ratio of number of frames, in which activities of machines are correctly recognized, to the total number of input frames. A larger accuracy stands for a better performance. In this paper, accuracy metric measures the overall performance of all activities.

$$
\text { Accuracy }=\frac{\text { Number of correct frames }}{\text { Total frames }}
$$

\footnotetext{
Row-normalized confusion matrix. Row-normalized confusion matrix summarizes the
} 
confusion between results generated by activity recognition methods and ground-truth labels of per-frame activity. When the original dataset has more than one type of activities, confusion matrix is a good way to imply the confusion between different activities. This matrix has been commonly used by other works dealing with construction resource activity recognition.

\subsubsection{Pose prediction metrics}

Two metrics are adopted to evaluate the performance of the pose prediction model based on machine keypoints, i.e. normalized error (NE) and percentage of correct keypoints (PCK). When calculating NE and PCK, we require a distance normalization parameter since bounding boxes of the detected machine will have different sizes in video frames. The distance normalization parameter $\left(s_{m}\right)$ is computed according to Equation (9).

$$
s_{m}=\max (h, w)
$$

where $h$ and $w$ is the height and width of the bounding box of an excavator in the $m^{\text {th }}$ frame, respectively.

Normalized error (NE). Normalized error (NE) is the average normalized distance between the forecasted keypoints and the actual values [48]. Only the keypoints included in the input frame (i.e. $v \geq 0$ ) are counted. A smaller NE suggests a better model performance. The calculation of $\mathrm{NE}$ is shown in Equation (10).

$$
N E=\frac{1}{M} \sum_{m=1}^{M} \frac{\sum_{k=1}^{K}\left(\frac{d_{m, k}}{s_{m}} \cdot \delta\left(v_{m, k} \geq 0\right)\right)}{\sum_{k=1}^{K} \delta\left(v_{m, k} \geq 0\right)}
$$

where $M$ is the total number of test images, $K$ is the total number of a piece of machine keypoints, $d_{m, k}$ is the Euclidean distance between the predicted position and the ground-truth label of the $k^{t h}$ keypoint in the $m^{t h}$ image, $s_{m}$ is the distance normalization parameter in the $m^{t h}$ image (see Equation (9)), and $v_{m, k}$ means the visibility of the $k^{t h}$ keypoint in the $m^{\text {th }}$ image.

Percentage of correct keypoints (PCK). PCK evaluates the accuracy by measuring the percentage of keypoints correctly forecasted over the total amount of keypoints in one category or the whole annotation set [49]. As shown in Equation (11), a candidate keypoint is assumed correctly located if its normalized error is smaller than a given threshold $\alpha$, where $\alpha \in[0,1]$. For human pose estimation and machine pose estimation, 0.05 is commonly used as the upper bound of the threshold $\alpha$. Therefore, $\operatorname{PCK}(\alpha=0.05)$ is adopted as an evaluation metric of machine pose prediction so as to assess the performance more precisely. 


$$
P C K(\alpha)=\frac{1}{M} \sum_{m=1}^{M} N E_{m}<\alpha, \alpha \in[0,0.05]
$$

where $\alpha$ is the threshold for calculating and plotting the PCK curves, and the definition of other parameters are illustrated in Equation (10).

\subsection{Experiment results}

\subsubsection{The results of the machine activity recognition module}

Three scenarios are experimented for investigating the effectiveness of pre-processing and postprocessing approaches. Table 2 shows the activity recognition accuracy for five videos and the final average accuracy under different scenarios. With the raw data, the proposed activity recognition methodology can only achieve an average accuracy of $44.79 \%$, probably because noises in the data and uncertainties of human operation severely mislead the proposed methodology and affect the recognition performance. In the second scenario, after applying wavelet transform pre-processing on the raw data, the results of the proposed activity recognition methodology are significantly improved with an average accuracy of $81.24 \%$. In the third scenario, we combine both the wavelet transform pre-processing and the rollback post-processing techniques to further enhance the performance of the proposed methodology. With such combination, the average accuracy reaches the highest value of $87.05 \%$, which indicates the effectiveness of the pre-processing and post-process techniques. In terms of the accuracy for each video, our activity recognition methodology achieves its best accuracy of $90.66 \%$ on video 152129 and the lowest accuracy of $84.81 \%$ on video 104154 . After investigating the dataset, we found that there were stones of different sizes in the area to be excavated in video 104154. In this case, it is necessary for the operator to operate the machine by intensively swinging the boom, arm and bucket of the excavator before "loading bucket" activity, which undoubtedly introduces more difficulties for the proposed activity recognition methodology to correctly categorize activity types. In comparison, in the last three videos (i.e. 150625, 152129, and 142932), excavators are operated more stably because the areas to excavate are filled with soils and small stones. Hence, poses of the target excavators changed smoothly following the certain pattern. In this way, there is not much influence on the sequential data of Body_end and Arm_bucket keypoints, which are most decisive keypoints in the proposed intuitive activity recognition methodology. But there are still some false activity recognition results in the last three videos. After investigating the videos, the authors find that most false activity recognition results occur in some short periods when the machine activity is transforming from one to another. During such short transition period, construction machines may exhibit very similar gestures with minor variations, making it hard to differentiate. In addition, algorithms for smoothening the collected signals would blur the boundaries between two consecutive activities. Therefore, the activity recognition methodology is likely to be misled and generate some false activity recognition results. Example results of correct and incorrect machine activity recognition are represented in Figure 11. 
Table 2. Accuracy of the machine activity recognition module

\begin{tabular}{cccc}
\hline Video ID & Raw data & $\begin{array}{c}\text { Wavelet transform } \\
\text { pre-processing }\end{array}$ & $\begin{array}{c}\text { Wavelet transform pre-processing } \\
\text { and rollback post-processing }\end{array}$ \\
\hline 104154 & $44.43 \%$ & $84.63 \%$ & $84.81 \%$ \\
134516 & $40.04 \%$ & $76.57 \%$ & $85.33 \%$ \\
150625 & $39.73 \%$ & $88.49 \%$ & $89.25 \%$ \\
152129 & $41.89 \%$ & $90.40 \%$ & $90.66 \%$ \\
142932 & $56.94 \%$ & $88.47 \%$ & $90.36 \%$ \\
Average & $44.79 \%$ & $81.24 \%$ & $87.05 \%$ \\
\hline
\end{tabular}



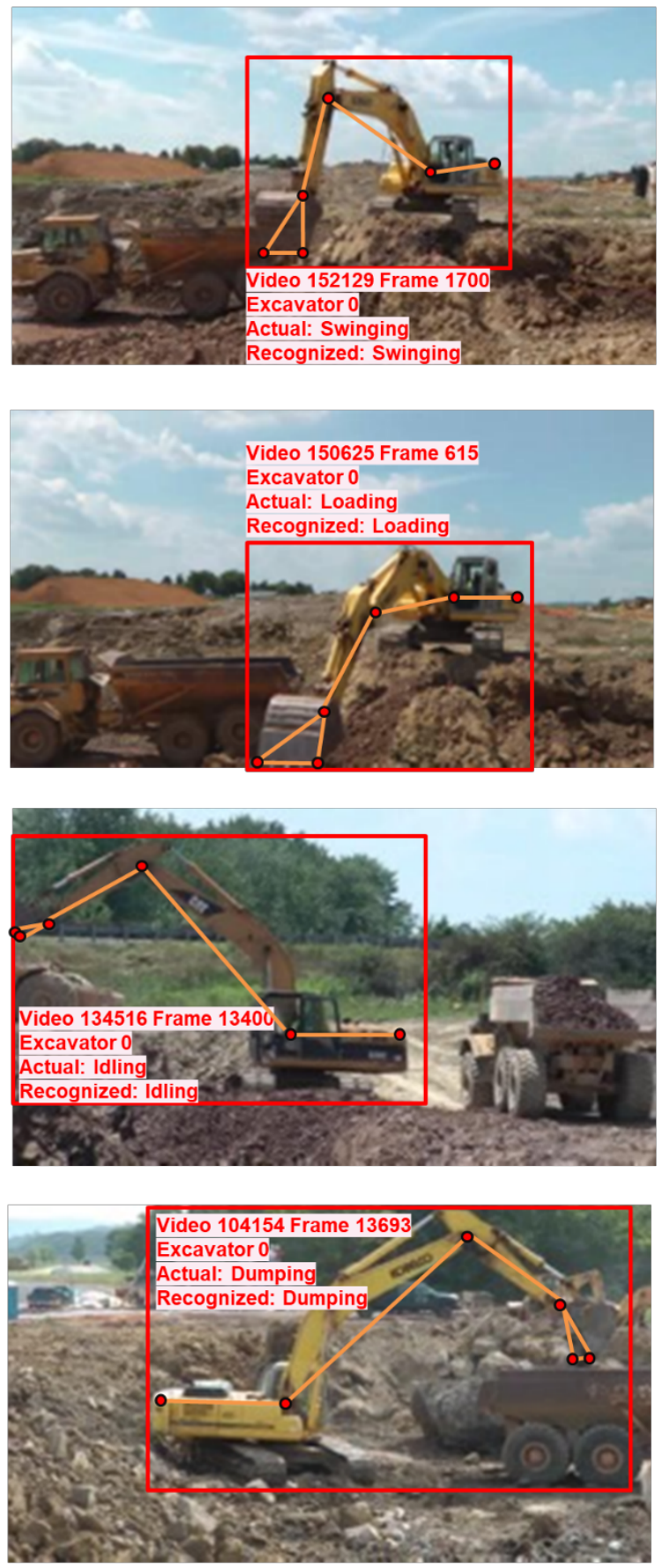

\section{Correct recognition result}
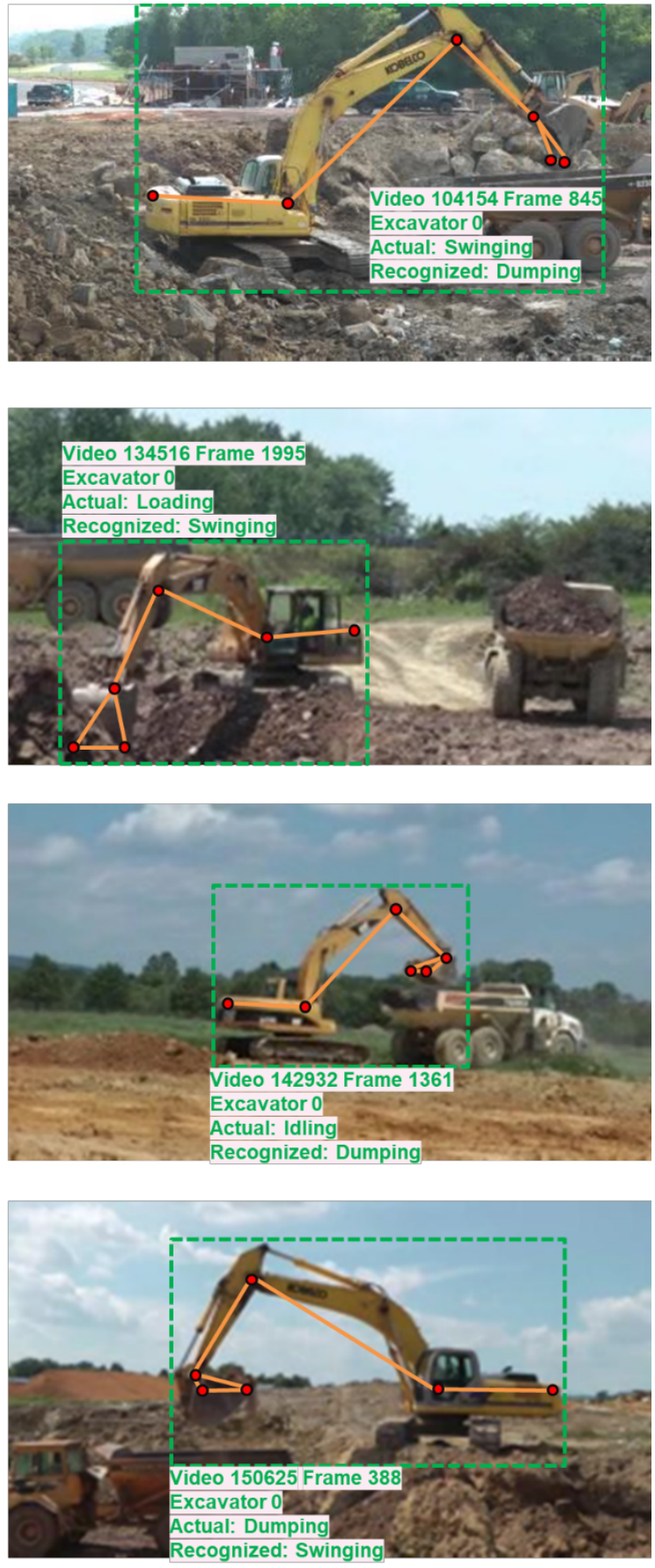

Incorrect recognition result

Figure 11. Example results of the machine activity recognition module

Apart from using accuracy to evaluate per-frame activity recognition results of all the available videos, the normalized confusion matrix is adopted to evaluate the performance of the proposed activity recognition methodology in terms of each atomic activity. The confusion matrix of the 
recognition results is drawn in Figure 12. It is noticed that the proposed methodology has very high performances in recognizing "dumping" and "loading bucket" activities without confusion between each other. However, our method confuses both "dumping" and "loading bucket" with the "swinging bucket" activity at a rate around $8 \%$. One reason can be that there is a gradual yet short transformation between two consecutive activities, which leads to the ambiguity for recognition. For example, at the moment when the excavator is changing from "dumping" to the "swinging bucket", it will show similar moving patterns to "swinging bucket". This reason also explains the observation that "swinging bucket" activities are confused with "loading bucket" and "dumping" at a rate of $10 \%$ and $9 \%$, respectively. Unlike excellent performances of recognizing "dumping", "loading bucket" and "swinging bucket" activities, the proposed methodology exhibits a relatively low performance when dealing with "idle" activities. According to Figure 12, our method easily confused "idle" activities with all other three activities. The rate of misclassifying "idle" as "dumping" activities by the proposed methodology is $4 \%$, which is relatively low, possibly because all the strict and intuitive criteria of deciding "dumping" activities must be met simultaneously, including (1) both a truck and an excavators co-exist; (2) the main body of the target excavator remains stationary; and (3) the bucket of the target excavator moves inside the dumping area. However, the proposed method is more likely to misclassify "idle" as "swinging bucket" or "loading bucket", with a much higher rate over $10 \%$. One reason could be that the excavator usually remains "idle", waiting for a full-loaded truck to be replaced by an empty one, and the excavator bucket stays outside the dumping area, which is similar to the situations defined for "swinging bucket" and "loading bucket". Additionally, the human operators, motion capture devices and data transmission process may introduce uncertain changes and unexpected noise to the motion data, which escalate recognition errors that "idle" is misclassified as "swinging bucket" or "loading bucket". Nevertheless, for safety management on construction sites, "idle" activities are less dangerous than other activities in the operational cycles. Therefore, to some extent, it is acceptable that "idle" activities are mis-recognized as "swinging bucket", "loading bucket" or "dumping" activities. 


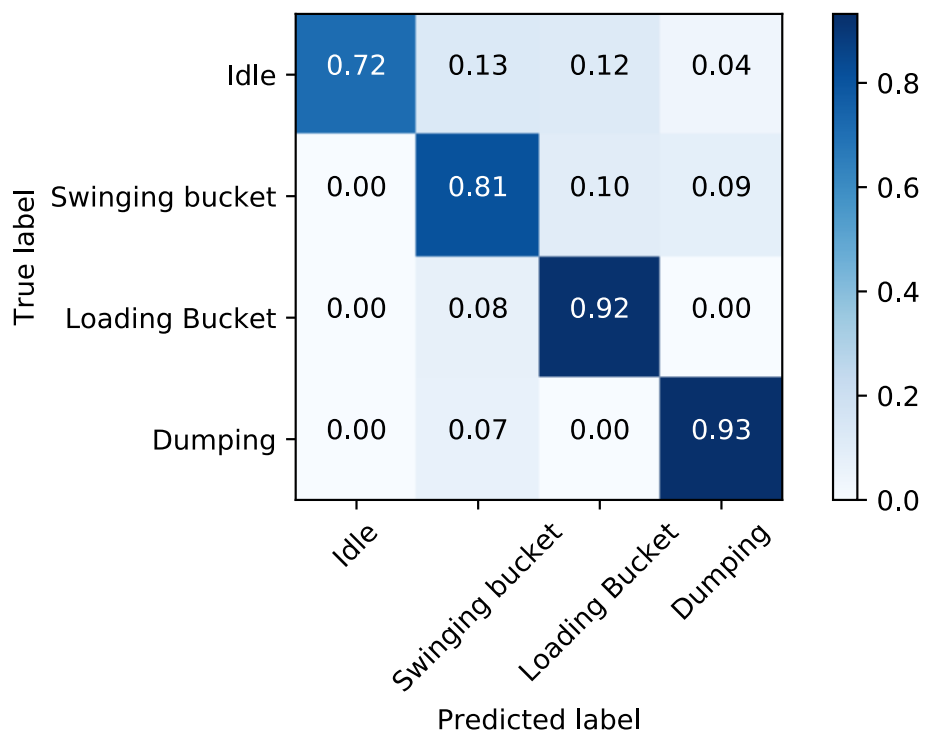

Figure 12. Normalized confusion matrix of the activity recognition results.

\subsubsection{The results of machine pose prediction module}

This section discusses the performance of the third module of the framework, machine pose prediction, individually. Therefore, we train, validate and test the motion prediction model in two settings, i.e. using keypoint feature vectors without or with actual historical activity information. The model performances with these two settings are compared using two evaluation metrics, i.e. normalized error (NE) and percentage of correct keypoints (PCK). Normalized error calculates the sum of errors from all predicted machine keypoints. A smaller NE value indicates a higher accuracy of the model.

As shown in Table 3, when the model is trained with the first setting, i.e. the activity data is not included in the input keypoint feature vectors, the proposed machine pose prediction model achieves an average normalized error of $24.59 \times 10^{-3}$ on all tested videos. With the second setting, the model is trained using keypoint feature vectors with the actual historical activities added, leading to an average NE value of $22.65 \times 10^{-3}$. Among all videos, the model generates the smallest $\mathrm{NE}$ value on video 104154, and the largest one on video 150625 , in both settings of the input keypoint feature vectors. In terms of the average NE of all the videos, input features with actual activity information significantly enhanced the performance of our proposed model with a NE reduction rate of $7.89 \%$. For each video, additional historical activity data improve the results of machine pose prediction model to different degrees. Specifically, the largest NE reduction is observed at video 142932 , with a reduction rate of $17.13 \%$, while the smallest one shows at video 150625 with a slight reduction of $0.71 \%$. The performance improvements due to adding historical activity data into input are more than $8 \%$ for the rest videos. Example results of the machine pose prediction are depicted in Figure 13, which illustrates the predicted excavator poses in 10 frames following a given frame in Video 150625. 


\begin{tabular}{cccc}
\hline \multirow{2}{*}{ Video ID } & Without Activity $\left(10^{-3}\right)$ & With Actual Activity $\left(10^{-3}\right)$ & NE Reduction \\
\hline 104154 & 16.76 & 15.33 & $8.53 \%$ \\
134516 & 24.54 & 22.25 & $9.33 \%$ \\
150625 & 36.81 & 36.55 & $0.71 \%$ \\
152129 & 23.84 & 21.69 & $9.02 \%$ \\
142932 & 21.02 & 17.42 & $\mathbf{1 7 . 1 3 \%}$ \\
Average & 24.59 & 22.65 & $7.89 \%$ \\
\hline
\end{tabular}

691

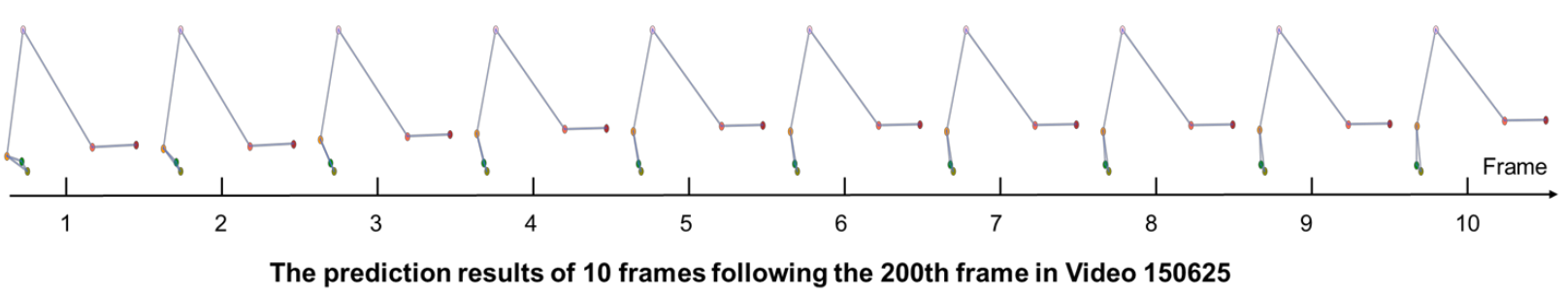

Figure 13. Example results of the machine pose prediction module

Apart from NE, PCK is computed to describe the percentage of keypoints in a future period that are correctly predicted by our machine pose prediction model. PCK requires a threshold during calculation and treats a machine pose keypoint as correctly predicted if the distance between the predicted and the ground-truth keypoint is smaller than the given threshold. Different from NE that focuses on the global performance improvement, PCK is only concerned about a part of keypoints that meet with precision requirements. A smaller threshold indicates it is more difficult for keypoints to be counted as correctly predicted. As mentioned in Section 4.3.2, we use PCK with a threshold $\alpha$ of 0.05 (i.e. $\operatorname{PCK}(\alpha=0.05)$ ) as one of our evaluation metrics. Overall, the model trained using keypoint features with actual activity information improves the PCK results with a $2.45 \%$ increase from $88.23 \%$ to $90.39 \%$, as shown in Table 4 . On the basis of the comparison of PCK values for different videos, an identical conclusion is drawn that the model generates the best and worst PCK on video 104154 and 150625, respectively. Yet the insight of PCK improvement is slightly different. By adopting information provided by historical activities in the input keypoint feature vectors, the video 142932 gains the largest PCK improvement of $6.27 \%$ at a threshold of 0.05 . However, there is not much increase in PCK values for video 104154 and 134516 , only with less than $1 \%$ of PCK improvement. The results of PCK improvement imply that the historical activity data, in general, can help the machine motion prediction model obtain more correct results when the threshold is as small as 0.05 , while the level of PCK improvement is different in terms of different site videos. A potential reason is that, apart from the historical activity data, the difference in machine operators, machine types, and site conditions will also affect the prediction. 
Table 4. Percentage of correct keypoints $(\operatorname{PCK}(\alpha=0.05))$ results of machine pose prediction

\begin{tabular}{cccc}
\hline \multirow{2}{*}{ Video ID } & Without Activity & With Actual Activity & PCK Improvement \\
\hline 104154 & $96.91 \%$ & $97.51 \%$ & $0.62 \%$ \\
134516 & $92.56 \%$ & $93.14 \%$ & $0.63 \%$ \\
150625 & $74.23 \%$ & $75.74 \%$ & $2.03 \%$ \\
152129 & $87.67 \%$ & $90.16 \%$ & $2.84 \%$ \\
142932 & $89.76 \%$ & $95.39 \%$ & $\mathbf{6 . 2 7 \%}$ \\
Average & $88.23 \%$ & $90.39 \%$ & $2.45 \%$ \\
\hline
\end{tabular}

It is commonly recognized that deep learning models, including RNN, can perform better when more training data are available. Therefore, it is reasonable that the GRU-based machine motion prediction method shows better performances in video 104154, which has the most frames as listed in Table 1. However, we noticed that video 134516 has a comparative number of frames but a worse pose prediction result. It is found that, compared to 104154 , video 134516 has a long period of "idle" state in the testing data, which is not witnessed in the training data. Similar to most deep learning models, testing on data with new patterns that not existing in training, can be one possible reason leading to the decreased performances of the GRU-based motion prediction methods. Among all the adopted videos, the number of frames of video 150625 is smallest, which brings more difficulties for the motion prediction model to effectively learning the historical moving patterns and accurately predicting the future pose of the target machine. Additionally, in video 150625 , the bucket of the target machine sometimes exceeds the monitoring scope, thereby exacerbating the inaccurate prediction and making its results the worst among all videos. Both NE and PCK results indicates that the actual activity data help video 142932 achieve the largest NE reduction and PCK improvements. We find that the excavation and dumping areas of the target construction machine in video 142932 are more fixed than those in other videos, which leads to clearer and more stable periodic patterns of the pose data when the target machine is performing different activities. In this case, the contextual clues from the actual activity data can help machine pose prediction more effectively. Based on the predicted poses of the machines, site managers are able to forecast the moving trends of the construction machines and more precisely to compute the potential danger zones around each construction machine. Through computing the spatial relationship between the target construction machine and other onsite entities such as workers and other machines, site managers can classify the situation of onsite construction-related entities into "normal", "danger now", and "potential danger", as described in [50]. In this way, pro-active safety warnings can be given to avoid potential hazards on construction sites.

\subsubsection{The performance of the overall framework}

In this section, we evaluate the performance of the overall framework by normalized error and percentage of correct keypoint (PCK) at a threshold of 0.05 , and the results are provided in Table 5. In general, the overall framework works well when integrating all independent modules and using output of the second module as input for the third module. The average normalized error of 
the overall framework is $22.71 \times 10^{-3}$ and the average PCK is $90.22 \%$. In terms of normalized error, the results of most tested videos are less than the average NE. The overall framework has the best results on video 104154 with a NE value of $15.39 \times 10^{-3}$, while has the worst NE value on video 150625 , which is $36.56 \times 10^{-3}$. It is noted that the results of $\operatorname{PCK}(\alpha=0.05)$ are larger than $85 \%$ for most videos. From the PCK values, we can draw similar conclusions regarding the best and the worst model performance as from the normalized error. In comparison with the motion prediction results from the individual third module trained with ground truth activity information, the overall framework generates slightly lower average performances in both NE and PCK using the activity recognition results from the second module as inputs for the last module. Such slight degraded performance of the framework is observed in the results of each tested video. This phenomenon is reasonable considering the proposed activity recognition module will result in cumulative errors, yet the results still demonstrate the relatively good performance of the whole framework for predicting machine poses.

Table 5. The performance of the overall framework

\begin{tabular}{ccc}
\hline Video ID & $\begin{array}{c}\text { Normalized error (NE) } \\
\left(10^{-3}\right)\end{array}$ & $\begin{array}{c}\text { Percentage of correct keypoints } \\
(\text { PCK }(\alpha=0.05))\end{array}$ \\
\hline 104154 & $\mathbf{1 5 . 3 9}$ & $\mathbf{9 7 . 4 4 \%}$ \\
134516 & 22.38 & $93.12 \%$ \\
150625 & $\mathbf{3 6 . 5 6}$ & $\mathbf{7 5 . 2 9 \%}$ \\
152129 & 21.76 & $89.94 \%$ \\
142932 & 17.46 & $95.33 \%$ \\
Average & 22.71 & $90.22 \%$ \\
\hline
\end{tabular}

\subsection{Discussion}

\subsubsection{Different data sources and representations}

Experiments have demonstrated that the proposed framework has good performances using motion data captured from surveillance videos. When the adopted on-site videos are captured from one common surveillance camera which can only provide $\mathrm{x}$ and $\mathrm{y}$ information with the value of $\mathrm{z}$ axis remaining the same (i.e. $\mathrm{z}=0$ in video data), $2 \mathrm{D}$ historical motion data of construction machines can be extracted by detection, tracking, pose estimation or other relevant algorithms. If simultaneous multi-camera video data are available, it is possible that $3 \mathrm{D}$ historical motion data can be constructed and studied by appropriate stereo vision techniques. Apart from surveillance cameras, many pre-installed devices such as GPS tags and IMUs are also potential tools for collecting historical motion data from on-site construction machines. It is noted that the activity recognition module considers the spatial relationships between construction machines in a very general way where the activity recognition methodology can be expressed in both $2 \mathrm{D}$ and $3 \mathrm{D}$. In addition, the machine pose prediction module is able to digest both $2 \mathrm{D}$ and $3 \mathrm{D}$ coordinates information. Therefore, it is feasible and applicable to realize both $2 \mathrm{D}$ and $3 \mathrm{D}$ motion pose prediction by the overall workflow of the proposed framework. 


\subsubsection{Machine activity recognition}

To enhance the performance of machine pose prediction by historical activities, this research proposes a module that recognizes activities performed by the target construction machine. It is known that many existing studies [30,51] in the field of machine activity recognition adopted black-box approaches, such as machine learning techniques, that are hard to explain the functional principles and require training on numerous labeled data with large computational cost. Unlike those studies, our activity recognition methodology is very simple and straightforward based on intuitive knowledge summarized from experiences of construction projects. With the help of wavelet transform pre-processing and rollback post-processing, the proposed activity recognition methodology achieves comparatively good performance for recognizing activities of excavators in long video streams without tedious pretraining on a lot of training data. By integrating prior knowledge such as working patterns and working natures, as well as other non-geometric construction information, the proposed intuitive and explainable activity recognition methodology for excavators is of high generalization capability, hence can also be developed for other construction machines.

\subsubsection{Machine motion prediction}

As the first work focusing on pose prediction of construction machines, the proposed framework contributes to filling the research gap with several benefits. Firstly, the proposed framework performs well in predicting short-term future poses in the coming $0.4 \mathrm{~s}$. During the training process, the proposed framework learns the movement trend of machine poses from the historical motion data by minimizing the error of all the pose keypoints during the period of $0.4 \mathrm{~s}$ simultaneously. Consequently, the proposed framework is meaningful and helpful that it can provide more response time for pro-active safety management. In addition to historical machine motions captured from geometry site information, the proposed framework considers semantic data obtained from non-geometric information (i.e. historical machine activities during earthmoving tasks in the validation experiments). According to the experiment results, the performance of machine motion prediction is enhanced when the activity information is added to the original keypoint features. The results indicate that, during machine motion prediction, we should not only focus on positions, velocities, and accelerations embedded in historical motion data, but also consider non-geometric data obtained from prior experiences and expert knowledge.

\section{Conclusion}

Safety is the first priority of all construction projects. Construction machines are the major source of safety issues on construction sites due to their frequent interactions with workers and other construction-related objects. Therefore, it is necessary to monitor states (i.e. locations, poses and movements) of construction machines for avoiding potential collisions and other accidents. Besides tracking the past and the current states of construction machines, evaluating the future states of machines should also be emphasized to inform site managers of potential on-site hazards and assist them to make appropriate arrangement for preventing accidents or severe loss.

This paper focuses on predicting future poses of construction machines based on historical motion data, which has been rarely studied so far. A framework is proposed for predicting construction machine poses considering both geometric and non-geometric construction information, which includes three modules: (1) machine motion capture, (2) machine activity 
recognition, and (3) machine pose prediction. The first module extracts historical motion data of construction machines from geometric construction information by processing surveillance videos and/or sensor signals. Based on the obtained motion data, the second module recognizes historical activities of machines through analyzing both geometric and non-geometric construction information. In the end, the last module generates future poses of construction machines by integrating historical machine poses and the outputs of the second module, i.e. machine activity information.

To validate the feasibility of the proposed framework, excavators are adopted as our experiment objects since they are the most complex construction machines with many deformable components and more variations in poses. Firstly, we create a motion capture dataset containing labels of machine locations and keypoints with various construction site scenes. Based on the observed working patterns and interaction characteristics among machines, an intuitive activity recognition methodology is developed, which does not require pretraining with a large amount of data. The average recognition accuracy is greatly increased to $87.05 \%$, after applying wavelet transform and rollback mechanism to reduce data noise and human operation uncertainties. It indicates that when the input data are clean and there is not much uncertainty, intuitive knowledge of construction projects is helpful for recognizing machine activities in a simple yet effective way. As for the third module, a GRU-based method is proposed for predicting machine poses in the future 10 frames. It is found that the performance of the individual pose prediction model is improved, with $7.89 \%$ reduction in NE value and $2.45 \%$ increase in PCK, after adding the activity information into the input keypoint features. Such result proves that non-geometric construction information can provide useful semantic clues to enhance machine pose prediction. In the end, we evaluate the whole framework by using the recognition results from the second module and the original motion data as the inputs for the prediction model. The whole framework achieves relatively good performance with an average NE of $22.71 \times 10^{-3}$ and an average PCK of $90.22 \%$, regardless of the potential accumulative errors from different modules. The results demonstrate the high potential of our proposed overall framework for predicting future poses of construction machines.

Therefore, the major contribution of this work is that it proposes a generic framework for predicting construction machine poses and improves the prediction performance by incorporating both geometric and non-geometric construction information. This work also reveals the potential of deep learning for interpreting complex construction site data. Besides, the proposed framework can be quite flexible, considering that various methods can be explored for the activity recognition module and the pose prediction module based on available motion data. In terms of practical application, this study contributes by assisting site managers to predict the motion of machines such as to more precisely compute the potential position and range of danger zones on construction sites. The predicted dander zones can help understand safety conditions of workers, proactively give safety warnings and thereby reduce potential interactive hazards on construction sites. Despite the good performance of our framework, there are still some limitations to be addressed. Our intuitive activity recognition methodology is applicable based on either 2D or 3D motion capture data. When motion data are obtained from surveillance videos, different camera views require different mapping matrix from global coordinates to image coordinates, and sometimes the captured videos will suffer from various levels of occlusions. Therefore, viewpoints of on-site cameras need to be carefully considered when the activity recognition methodology is adopted with new settings of surveillance cameras or in new construction sites. Another limitation is that we directly use a basic GRU network for the machine motion prediction module. Although the 
GRU network validates the feasibility and applicability of the proposed framework, new architectures still need to be developed for improving the performance of machine motion prediction. In the future, we will look into each module of the proposed framework with the hope of acquiring better performances.

\section{Acknowledgments}

Financial supports of this study by the Hong Kong PhD Fellowship Scheme (HKPFS) to Han LUO and Peter K. Y. WONG are gratefully acknowledged.

\section{References}

[1] U.S. Bureau of Labor Statistics (BLS), National census of fatal occupational injuries in 2017, 2018. www.bls.gov/iif/oshcfoil.htm (accessed May 20, 2018).

[2] MOHURD, Report of safety accidents in China's building construction activities in 2017, 2018. http://www.mohurd.gov.cn/wjfb/201803/t20180322_235474.html (accessed May 20, 2018).

[3] Hong Kong Labour Department, Occupational Safety and Health Statistics 2017, 2018. https://www.labour.gov.hk/eng/osh/content10.htm (accessed May 20, 2018).

[4] M. Memarzadeh, M. Golparvar-Fard, J.C. Niebles, Automated 2D detection of construction equipment and workers from site video streams using histograms of oriented gradients and colors, Automation in Construction. 32 (2013) 24-37. https://doi.org/10.1016/j.autcon.2012.12.002.

[5] M. Golparvar-Fard, A. Heydarian, J.C. Niebles, Vision-based action recognition of earthmoving equipment using spatio-temporal features and support vector machine classifiers, Advanced Engineering Informatics. 27 (2013) 652-663. https://doi.org/10.1016/J.AEI.2013.09.001.

[6] H. Kim, H. Kim, Y.W. Hong, H. Byun, Detecting construction equipment using a regionbased fully convolutional network and transfer learning, Journal of Computing in Civil Engineering. 32 (2018). https://doi.org/10.1061/(ASCE)CP.1943-5487.0000731.

[7] M.M. Soltani, Z. Zhu, A. Hammad, Skeleton estimation of excavator by detecting its parts, Automation in Construction. 82 (2017) 1-15. https://doi.org/10.1016/j.autcon.2017.06.023.

[8] C.-J. Liang, K.M. Lundeen, W. McGee, C.C. Menassa, S. Lee, V.R. Kamat, Stacked hourglass networks for markerless pose estimation of articulated construction robots, in: Proceedings of the 35th International Symposium on Automation and Robotics in Construction (ISARC), 2018. https://doi.org/10.22260/isarc2018/0120.

[9] H. Luo, M. Wang, P.K.Y. Wong, J.C.P. Cheng, Full body pose estimation of construction equipment using computer vision and deep learning techniques, Automation in Construction. 110 (2020) 103016. https://doi.org/10.1016/j.autcon.2019.103016.

[10] C. Zhang, Improving crane safety by agent-based dynamic motion planning using UWB real-time location system, Concordia University, 2010. https://spectrum.library.concordia.ca/7177/ (accessed April 13, 2019). 
[11] H.K. Chiu, E. Adeli, B. Wang, D.A. Huang, J.C. Niebles, Action-agnostic human pose forecasting, in: Proceedings - 2019 IEEE Winter Conference on Applications of Computer Vision, WACV 2019, 2019: pp. 1423-1432. https://doi.org/10.1109/WACV.2019.00156.

[12] M.M. Soltani, Z. Zhu, A. Hammad, Framework for location data fusion and pose estimation of excavators using stereo vision, Journal of Computing in Civil Engineering. 32 (2018) 04018045. https://doi.org/10.1061/(asce)cp.1943-5487.0000783.

[13] S. Chae, T. Yoshida, Application of RFID technology to prevention of collision accident with heavy equipment, Automation in Construction. 19 (2010) 368-374. https://doi.org/10.1016/j.autcon.2009.12.008.

[14] H. Vogt, Efficient object identification with passive RFID tags, in: Lecture Notes in Computer Science (Including Subseries Lecture Notes in Artificial Intelligence and Lecture Notes in Bioinformatics), 2002: pp. 98-113. https://doi.org/10.1007/3-540-4586629.

[15] T. Cheng, M. Venugopal, J. Teizer, P.A. Vela, Performance evaluation of ultra wideband technology for construction resource location tracking in harsh environments, Automation in Construction. 20 (2011) 1173-1184. https://doi.org/10.1016/J.AUTCON.2011.05.001.

[16] S. Chi, C.H. Caldas, Automated object identification using optical video cameras on construction sites, Computer-Aided Civil and Infrastructure Engineering. 26 (2011) 368380. https://doi.org/10.1111/j.1467-8667.2010.00690.x.

[17] S. Arabi, A. Haghighat, A. Sharma, A deep-learning-based computer vision solution for construction vehicle detection, Computer-Aided Civil and Infrastructure Engineering. (2020) mice.12530. https://doi.org/10.1111/mice.12530.

[18] F.A. Bender, S. Goltz, T. Braunl, O. Sawodny, Modeling and offset-free model predictive control of a hydraulic mini excavator, IEEE Transactions on Automation Science and Engineering. 14 (2017) 1682-1694. https://doi.org/10.1109/TASE.2017.2700407.

[19] S. Lee, M.-S. Kang, D.-S. Shin, C.-S. Han, Estimation with applications to dynamic status of an excavator without renovation, in: Proceedings of the International Symposium on Automation and Robotics in Construction (ISARC), 2012. https://doi.org/10.22260/ISARC2012/0093.

[20] H. Kim, C.R. Ahn, D. Engelhaupt, S. Lee, Application of dynamic time warping to the recognition of mixed equipment activities in cycle time measurement, Automation in Construction. 87 (2018) 225-234. https://doi.org/10.1016/J.AUTCON.2017.12.014.

[21] F. Ghassemi, S. Tafazoli, P.D. Lawrence, K. Hashtrudi-Zaad, An accelerometer-based joint angle sensor for heavy-duty manipulators, in: Proceedings 2002 IEEE International Conference on Robotics and Automation (Cat. No.02CH37292), IEEE, 2002: pp. 17711776. https://doi.org/10.1109/ROBOT.2002.1014798.

[22] P. Cheng, B. Oelmann, Joint-angle measurement using accelerometers and gyroscopes - A survey, IEEE Transactions on Instrumentation and Measurement. 59 (2010) 404-414. https://doi.org/10.1109/TIM.2009.2024367.

[23] Y. Li, C. Liu, Integrating field data and 3D simulation for tower crane activity monitoring and alarming, Automation in Construction. 27 (2012) 111-119. https://doi.org/10.1016/j.autcon.2012.05.003.

[24] Trimble, GCSFlex. https://www.trimble.com/construction/heavy-civil/machinecontrol/FlexFamily/GCSFlexExc.aspx?dtID=overview\& (accessed April 20, 2011).

[25] Leica, Leica iCON iXE3 - 3D System. https://leica-geosystems.com/products/machinecontrol-systems/excavator (accessed April 10, 2017). 
[26] F. Vahdatikhaki, A. Hammad, H. Siddiqui, Optimization-based excavator pose estimation using real-time location systems, Automation in Construction. 56 (2015) 76-92. https://doi.org/10.1016/J.AUTCON.2015.03.006.

[27] M. Haga, W. Hiroshi, K. Fujishima, Digging control system for hydraulic excavator, Mechatronics. 11 (2001) 665-676. https://doi.org/10.1016/S0957-4158(00)00043-X.

[28] Z. Li, X. Li, S. Liu, L. Jin, A study on trajectory planning of hydraulic robotic excavator based on movement stability, in: 2016 13th International Conference on Ubiquitous Robots and Ambient Intelligence, URAI 2016, Institute of Electrical and Electronics Engineers Inc., 2016: pp. 582-586. https://doi.org/10.1109/URAI.2016.7625784.

[29] R. Akhavian, A.H. Behzadan, Construction equipment activity recognition for simulation input modeling using mobile sensors and machine learning classifiers, Advanced Engineering Informatics. 29 (2015) 867-877. https://doi.org/10.1016/j.aei.2015.03.001.

[30] J. Kim, S. Chi, Action recognition of earthmoving excavators based on sequential pattern analysis of visual features and operation cycles, Automation in Construction. 104 (2019) 255-264. https://doi.org/10.1016/J.AUTCON.2019.03.025.

[31] D. Roberts, M. Golparvar-Fard, End-to-end vision-based detection, tracking and activity analysis of earthmoving equipment filmed at ground level, Automation in Construction. 105 (2019) 102811. https://doi.org/10.1016/J.AUTCON.2019.04.006.

[32] J. Kim, S. Chi, J. Seo, Interaction analysis for vision-based activity identification of earthmoving excavators and dump trucks, Automation in Construction. 87 (2018) 297308. https://doi.org/10.1016/J.AUTCON.2017.12.016.

[33] R.E. Kalman, R.S. Bucy, New results in linear filtering and prediction theory, Journal of Fluids Engineering, Transactions of the ASME. 83 (1961) 95-108. https://doi.org/10.1115/1.3658902.

[34] D. Vasquez, T. Fraichard, Motion prediction for moving objects: A statistical approach, in: Proceedings - IEEE International Conference on Robotics and Automation, 2004: pp. 3931-3936. https://doi.org/10.1109/robot.2004.1308883.

[35] M. Shan, S. Worrall, E. Nebot, Long term vehicle motion prediction and tracking in large environments, in: IEEE Conference on Intelligent Transportation Systems, Proceedings, ITSC, 2011: pp. 1978-1983. https://doi.org/10.1109/ITSC.2011.6082922.

[36] L. Sun, Z. Yan, S.M. Mellado, M. Hanheide, T. Duckett, 3DOF pedestrian trajectory prediction learned from long-term autonomous mobile robot deployment data, in: Proceedings - IEEE International Conference on Robotics and Automation, Institute of Electrical and Electronics Engineers Inc., 2018: pp. 5942-5948. https://doi.org/10.1109/ICRA.2018.8461228.

[37] J. Bütepage, M.J. Black, D. Kragic, H. Kjellström, Deep representation learning for human motion prediction and classification, in: Proceedings - 30th IEEE Conference on Computer Vision and Pattern Recognition, CVPR 2017, 2017: pp. 1591-1599. https://doi.org/10.1109/CVPR.2017.173.

[38] S. Ren, K. He, R. Girshick, J. Sun, Faster R-CNN: Towards real-time object detection with region proposal networks, IEEE Transactions on Pattern Analysis and Machine Intelligence. 39 (2017) 1137-1149. https://doi.org/10.1109/TPAMI.2016.2577031.

[39] Z. Kalal, K. Mikolajczyk, J. Matas, Tracking-learning-detection, IEEE Transactions on Pattern Analysis and Machine Intelligence. 34 (2012) 1409-1422. https://doi.org/10.1109/TPAMI.2011.239. 
[40] Y. Chen, Z. Wang, Y. Peng, Z. Zhang, G. Yu, J. Sun, Cascaded Pyramid Network for multi-person pose estimation, in: 2018 IEEE/CVF Conference on Computer Vision and Pattern Recognition, IEEE, 2018: pp. 7103-7112. https://doi.org/10.1109/CVPR.2018.00742.

[41] J. Yoon, J. Kim, J. Seo, S. Suh, Spatial factors affecting the loading efficiency of excavators, Automation in Construction. 48 (2014) 97-106. https://doi.org/10.1016/j.autcon.2014.08.002.

[42] Y. Xu, J.B. Weaver, D.M. Healy, J. Lu, Wavelet transform domain filters: a spatially selective noise filtration technique, IEEE Transactions on Image Processing. 3 (1994) 747-758. https://doi.org/10.1109/83.336245.

[43] K. Cho, B. Van Merriënboer, C. Gulcehre, D. Bahdanau, F. Bougares, H. Schwenk, Y. Bengio, Learning phrase representations using RNN encoder-decoder for statistical machine translation, in: EMNLP 2014 - 2014 Conference on Empirical Methods in Natural Language Processing, Proceedings of the Conference, Association for Computational Linguistics (ACL), 2014: pp. 1724-1734. https://doi.org/10.3115/v1/d141179 .

[44] J. Chung, C. Gulcehre, K. Cho, Y. Bengio, Empirical evaluation of gated recurrent neural networks on sequence modeling, (2014). http://arxiv.org/abs/1412.3555.

[45] OpenCV Team, Computer vision annotation tool (CVAT). https://github.com/opencv/cvat (accessed December 29, 2018).

[46] A. Paszke, S. Gross, S. Chintala, G. Chanan, E. Yang, Z. DeVito, Z. Lin, A. Desmaison, L. Antiga, A. Lerer, Automatic differentiation in PyTorch, in: NIPS 2017 Workshop Autodiff Decision Program Chairs, 2017: pp. 2-8. https://doi.org/10.1145/24680.24681.

[47] D.P. Kingma, J. Ba, Adam: a method for stochastic optimization, in: The 3rd International Conference for Learning Representations, San Diego, 2014. https://arxiv.org/pdf/1412.6980.pdf.

[48] X. Zou, X. Kong, W. Wong, C. Wang, Y. Liu, Y. Cao, FashionAI: A hierarchical dataset for fashion understanding, in: IEEE Computer Society Conference on Computer Vision and Pattern Recognition Workshops, 2019: pp. 296-304. https://doi.org/10.1109/CVPRW.2019.00039.

[49] M. Andriluka, L. Pishchulin, P. Gehler, B. Schiele, 2D Human Pose Estimation: New Benchmark and State of the Art Analysis, in: 2014 IEEE Conference on Computer Vision and Pattern Recognition, IEEE, 2014: pp. 3686-3693. https://doi.org/10.1109/CVPR.2014.471.

[50] M. Wang, P. Wong, H. Luo, S. Kumar, V. Delhi, J. Cheng, Predicting safety hazards among construction workers and equipment using computer vision and deep learning techniques, in: Proceedings of the 36th International Symposium on Automation and Robotics in Construction, ISARC 2019, 2019: pp. 399-406. https://doi.org/10.22260/isarc2019/0054.

[51] K.M. Rashid, J. Louis, Times-series data augmentation and deep learning for construction equipment activity recognition, Advanced Engineering Informatics. 42 (2019) 100944. https://doi.org/10.1016/j.aei.2019.100944. 